\title{
Oxidative Medicine and Cellular Longevity Hsa_circ_0013731 mediated by E2F1 inhibits ferroptosis in hepatocellular carcinoma cells by sponging miR-877-3p and targeting SLC7A11
}

\section{Shiji Fang}

Lishui Hospital of Zhejiang University

\section{Weiqian Chen}

Lishui Hospital of Zhejiang University Jiayi Ding

Lishui Hospital of Zhejiang University

Dengke Zhang

Lishui Hospital of Zhejiang University

Liyun Zheng

Lishui Hospital of Zhejiang University Jingjing Song

Lishui Hospital of Zhejiang University

Jianfei Tu

Lishui Hospital of Zhejiang University

\section{Zhongwei Zhao}

Lishui Hospital of Zhejiang University

\section{Rongfang Qiu}

Lishui Hospital of Zhejiang University

Qiaoyou Weng

Lishui Hospital of Zhejiang University

Chenying Lu

Lishui Hospital of Zhejiang University

Yang Yang

Lishui Hospital of Zhejiang University Jiansong Ji ( $\nabla_{\text {ji_j_s@sina.com ) }}$

Lishui Hospital of Zhejiang University

Research 
Keywords: hepatocellular carcinoma, circ_0013731, miR-877-3p, ferroptosis

Posted Date: August 5th, 2021

DOI: https://doi.org/10.21203/rs.3.rs-40234/v2

License: (c) (1) This work is licensed under a Creative Commons Attribution 4.0 International License. Read Full License 


\section{Abstract}

Background: The regulatory loop between circular RNAs and microRNAs has a vital role in cell death. Ferroptosis is the form of iron-dependent cell death, which is distinct from necroptosis and apoptosis. Increasing evidences showed that ferroptosis is an important form of cell death in hepatocellular carcinoma.

Methods: Real-time PCR were used to examine the expression level of circ_0013731 in hepatocellular carcinoma tissues. Edu and colony formation were performed to detect the cell proliferation. A luciferase reporter assay was used to determine the relationship between circ_0013731, miR-877-3p and SLC7A11. ChIP-qPCR assays were performed to examine the potential binding of E2F1 to the circ_0013731 promoter. Iron Assay Kit (Sigma Aldrich) was used to detect total iron or Fe ${ }^{2+}$. C11 BODIPY 581/591 staining and flow cytometer were used to examine the Lipid ROS. The role of circ_0013731 was examined in xenograft tumors model.

Results: We revealed that the expression level of circ_0013731 was elevated in hepatocellular carcinoma. Moreover, E2F1 promote the transcription of circ_0013731. Overexpression of circ_0013731 promoted cell growth and inhibited ferroptosis in SMMC-7721 and QGY-7703 in vitro. miR-877-3p was proved as the direct target of circ_0013731. Then, inhibition of miR-877-3p enhanced cell growth and inhibited ferroptosis. Further mechanism research demonstrated that circ_0013731 upregulated the expression level of SLC7A11 by sponging miR-877-3p. Finally, circ_0013731 promoted HCC growth via miR-877-3p/ SLC7A11 axis in vivo.

Conclusions: Our data reveal that circ_0013731 mediated by E2F1 facilitates cell growth and suppressed the ferroptosis via miR-877-3p/ SLC7A11 axis in hepatocellular carcinoma. Therefore, circ_0013731 could be acted as potential therapeutical target for hepatocellular carcinoma.

\section{Background}

Hepatocellular carcinoma (HCC) is a lethal digestive malignancy worldwide and it is considered the second cause of cancer-related death [1, 2]. The current problem is that HCC still has high recurrence and mortality rate, even though patients received standardized treatment including operation, chemotherapy and radiotherapy [3]. Moreover, a part of patients are usually diagnosed at late stages, thus missing the time window for radical cure [4]. Thus, finding a novel target for treatment is vital for curing of HCC.

CircRNAs are non-coding RNAs with closed loop and they are lack of 5' and $3^{\prime}$ polarity and a polyadenylated tail $[5,6]$. The different roles of circRNAs have been reported in HCC. circRNA_101237 is acted as a vital prognostic biomarker for HCC [7]. circRNA_104075 promotes YAP-dependent HCC progression via bounding to HNF4a [8].

circRNA_cSMARCA5 suppresses metastasis by upregulation of TIMP3 in HCC [9]. Based on circBase (http://www.circbase.org/), circ_0013731 (chr1:117944807-117945063) was translated from exon2 of 
MAN1A2 by back-splicing (256 bp). circ_0013731 was upregulated in triple-negative breast cancer, but its role in tumor is still unknown.

MicroRNAs (miRNAs), small noncoding RNAs of 20-25 nucleotides in length, regulate the expression of downstream targets by post-transcriptional modulation $[10,11]$. CircRNAs serve as molecular sponge and adsorbed miRNAs abiding by base complementary pairing principle [12, 13]. circTRIM33-12 in HCC inhibits the immune evasion by sponging miR-191 and releasing TET1 [14]. Overexpression of circMT01 facilitates HCC tumorigenesis by sponging miR-9 and downregulating p21 expression [15]. Circ_0001955 in HCC up-regulates TRAF6 and MAPK11 expression, the target of miR-516a-5p, resulting in promotion of cell invasion [16]. miR-877-3p was identified as a novel anti-tumor regulator in malignant tumor. miR-877$3 p$ in gastric cancer inhibited metastasis by targeting VEGFA [17]. miR-877-3p suppressed the cell proliferation in bladder cancer [18]. However, the role of miR-877-3p in ferroptosis is largely unclarified.

The reprogramming of cell death is vital for HCC progression. The great progress has been achieved in necrotic cell death and apoptotic $[19,20]$. Ferroptosis is the form of iron-dependent cell death, which is distinct from necroptosis and apoptosis [21]. In this study, we found circ_0013731 was elevated in HCC and mediated by E2F1. Moreover, circ_0013731 facilitates cell growth and inhibited ferroptosis in HCC. Furthermore, we found that circ_0013731 suppressed ferroptosis through miR-877-3p /SLC7A11 axis. Finally, we elaborated circ_0013731 promoted HCC growth and regulated miR-877-3p/ SLC7A11 axis in vivo.

\section{Materials And Methods}

\section{Cell culture}

The HCC lines, namely, SMMC-7721, QGY-7703 and Huh7 were purchased from Academy of Sciences of Shanghai (Shanghai, China). $\mathrm{HCC}$ lines were maintained at $37^{\circ} \mathrm{C}$ with $5 \% \mathrm{CO}_{2}$ and cultured in RPMI 1640 medium (Gibco, Carlsbad, CA, USA) supplemented with 10\% fetal bovine serum (Gibco, Carlsbad, CA, USA).

\section{Tissue samples}

45 HCC tissues were enrolled in this study. HCC diagnosis was based on histopathology. The study was carried out under the approval by the Ethics Committee of Lishui Hospital of Zhejiang University. Participants all provided their informed written consent.

\section{EdU assay}

EdU assay was performed in HCC cells. Briefly, HCC cells were plated into 96-well plates. HCC cells were cultured for 2 hours after treated with $50 \mu \mathrm{M}$ EdU. Cells were fixed with $4 \%$ paraformaldehyde then 
stained by Hoechst 33342 and Apollp reaction cocktail. Results were counted five random fields by using a fluorescence microscopy.

\section{Cell transfection}

LV- circ_0013731 and sh- circ_0013731 were purchased from GeneCreate Biological Engineering Co., Ltd. (China). Mimic- miR-877-3p or inhibition-miR-877-3p was purchased from (RiboBio Corporation, Guangzhou, China). Cells were transfected with LV/sh- circ_0013731 or mimic/inhibitor-miR-877-3p by using Lipofectamine 3000 (Invitrogen, Carlsbad, CA, USA).

\section{qRT-PCR}

Total RNA was obtained using TRIzol reagent. RT-PCR was conducted using a SYBR Premix Ex Taq (TaKaRa, Dalian, China). Relative expression of genes was calculated by $2^{-\triangle \triangle C T}$.and SLC7A11, GAPDH and Tubulin expression levels were examined using the following specific primers:

F-5'TCTCCAAAGGAGGTTACCTGC3' and

R-5' AGACTCCCCTCAGTAAAGTGAC3';

F-5'GGAGCGAGATCCCTCCAAAAT3' and

R-5'GGCTGTTGTCATACTTCTCATGG3';

F-5' GGCCAAGGGTCACTACACG3' and

R-5' GCAGTCGCAGTTTTCACACTC3'.

\section{Western blot}

HCC cells and tissues were lysed and protein concentration of total cell lysates was tested by the BCA assay. Protein samples (25-30ug) were loaded to electrophoresis and then transferred to PVDF membrane. After blocked in 5.5\% non-fat milk for 2 hours, primary antibodies were used for incubation. Specific primary antibodies were used in this research included anti-SLC7A11, anti-GAPDH and antiTubulin (Abcam, Cambridge, UK, 1:1000) for overnight at $4^{\circ} \mathrm{C}$. Finally, the membrane was analyzed by the QuantityOne software (BioRad, Hercules, CA, USA).

\section{Luciferase activity assay}

The potential putative sequences of the binding site in MAN1A2 promoter/E2F1, circ_0013731/miR-877$3 p$ and miR-877-3p/ SLC7A11 and corresponding mutated sequences were cloned into pGL3 vector. 
About $1.8 \times 10^{5} \mathrm{HCC}$ cells were seeded in 48-well plate before transfection. After transfection with inhibitor-miR-877-3p, LV- circ_0013731 or corresponding negative control, the relative luciferase absorbance value of each group was examined by Dual-Luciferase Reporter Assay System (Promega, \#E1910).

\section{Immunofluorescent staining}

Histological section was fixed by cold acetone for $10 \mathrm{~min}$ and incubated in a 1\% BSA/PBS solution. Antibodies against SLC7A11 (1:100, Santa Cruz, Dallas, TX, USA) was used for incubation with section overnight at $4^{\circ} \mathrm{C}$. Next, secondary antibody (1:500, Santa Cruz, Dallas, TX, USA) were incubated with section. Wide-field fluorescence microscopy (Carl Zeiss, Jena, Germany) was used to visualize the slide.

\section{RNA Immunoprecipitation}

Magna RIP RNA Binding Protein Immunoprecipitation Kit was used for RNA Immunoprecipitation. HCC cell extract was incubated with RIPA buffer with magnetic beads conjugated with human Ago2 antibody for $9 \mathrm{~h}$. Normal IgG was used for negative control. Finally, immunoprecipitated RNA was collected and subjected to qRT-PCR analysis.

\section{Biotin labeled probe pull down assay}

HCC cells were lysed in lysis buffer. Next, $3 \mu \mathrm{g}$ biotin labeled probe was added to the buffer lysis and incubated for 4 hours at $25^{\circ} \mathrm{C}$. To pull down the circRNA-microRNA complex, strepavidin magnetic beads (Thermo Fisher) were added to the buffer and slowly rotated for another 4 hours. Then, washing step was carried out for 4 times. Finally, the binding RNA was extracted with TRIzol reagent and subjected to qRTPCR analysis.

\section{Lipid ROS assays}

Following instructions [22], HCC cells were treated by C11-BODIPY $(10 \mu \mathrm{M})$ and incubated for half hour. Afterward, washing step was performed twice times. C11-BODIPY was removed by cold PBS. The fluorescence of C11-BODIPY581 was examined by the simultaneous acquisition of green $(484 / 510 \mathrm{~nm})$ and red signals $(581 / 610 \mathrm{~nm})$ using a flow cytometer.

\section{Iron assays}

Following instructions [23], Iron Assay Kit (Sigma Aldrich) was used to detect total iron or $\mathrm{Fe}^{2+}$ in $\mathrm{HCC}$. Following instructions, Iron Assay buffer and Iron Reducer were added in turn to the samples. In dark 
conditions, samples were mixed well and incubated for half hour after adding Iron Reducer. Samples were incubated for one hour after adding Iron Probe. Lastly, the absorbance was examined at $593 \mathrm{~nm}$ (A593).

\section{In vivo study}

Female nude mice (5 weeks old) were purchased from Shanghai Vital River Animal Company. A circ_0013731 stable overexpression or knockout SMMC-7721 and QGY-7703 cell line was constructed by LV-circ_0013731 or sh-circ_0013731. Xenograft tumors model were established by subcutaneous transplantation. For the xenograft tumor model, $6.4 \times 10^{6}$ cells were subcutaneously injected into the left inguinal of nude mice. The volume of tumor was counted for $0,7,14,21,28$ days. Tumor weight was counted at 28 days. This work was under the Guide for the Care and Use of Laboratory Animals of the National Institutes of Health.

\section{Statistical analysis}

All the data were analyzed using SPSS software. Student $t$ test was used to compare the difference of

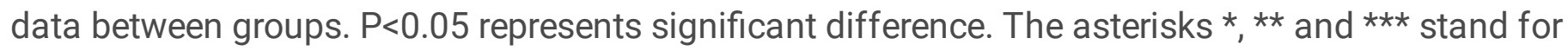
$p<0.05, p<0.01$ and $p<0.001$, respectively.

\section{Results}

\section{circ_0013731 was elevated in hepatocellular carcinoma}

To investigate the abundance of circ_0013731, RNase R digestion was used to identify circ_0013731 circular transcripts and MAN1A2 linear transcripts. Results shown that circ_0013731 was more resistant to RNase R digestion than MAN1A2 and GAPDH linear transcripts (Fig. 1a). circ_0013731 in HCC samples $(\mathrm{N}=45)$ showed an increased level compared with that in normal subjects as shown in Fig. 1b. Histogram and pie chart of the proportions of HCC samples in which circ_0013731 expression was downregulated $(9 / 45,20 \%$, blue), no change $(2 / 45,4.4 \%$, green), or upregulated $(34 / 45,75.6 \%$, red) as shown in Fig. 1 c. Then, the relative expression of circ_0013731 in primary tumor and portal vein tumor thrombus $(\mathrm{N}=26)$ was detected by qRT-PCR. Results showed that an increased level in portal vein tumor thrombus than that in primary tumor (Fig. 1d). As shown in Fig. 1e, the expression level of circ_0013731 was elevated in T3T4 subjects $(\mathrm{N}=30)$ than that in T1-2 subjects $(\mathrm{N}=15)$. Moreover, the expression level of circ_0013731 was upregulated in $\mathrm{N} 1$ samples ( $\mathrm{N}=11)$ than $\mathrm{N} 0$ samples ( $\mathrm{N}=34)$ (Fig. 1f). Finally, the expression level of circ_0013731 was also elevated in HCC cells (Fig. 19). Thus, the expression level of circ_0013731 was elevated in HCC.

\section{The expression of circ_0013731 was mediated by transcription factor E2F1}


E2F1 is a vital transcription factors and it can upregulate the circRNA expression at transcriptional level by binding to promoter. Based on circBase (http://www.circbase.org/), circ_0013731 was generated from MAN1A2 gene by back-splicing (256 bp). Four putative E2F1-binding sites on MAN1A2 promoter were found by bioinformatics prediction (https://www.gcbi.com.cn/gclib/html/index) and that of wild type or mutant promoter was established (Fig. 2a). Then, SMMC-7721 and QGY-7703 were transfected with pcDNA3.1-E2F1 or si-E2F1. The expression level of MAN1A2 was upregulated in pcDNA3.1-E2F1 group and was downregulated in si-E2F1 group (Fig. 2b-e). Then, correlation analysis revealed positive correlation between the levels of MAN1A2 and E2F1 mRNA in 45 HCC samples (Fig. 2f). Moreover, luciferase reporter assays were performed in SMMC-7721 and QGY-7703. Cells were transfected by pcDNA3.1-E2F1 or si-E2F1. Results showed that luciferase activity was elevated by overexpression of E2F1 and was decreased by knockdown of E2F1 (Fig. 2g-j). Afterwards, ChIP-qPCR assays were performed in SMMC-7721 and QGY-7703 cells. We proved that putative E2F1 binding site the MAN1A2 promoter was bound to in HCC cells, IgG was used as a negative control (Fig. $\mathbf{2 k}$, I). The expression levels of circ_0013731 in SMMC-7721 and QGY-7703 cells transfected with pcDNA3.1-E2F1 or si-E2F1 were assessed. circ_0013731 was positively regulated by pcDNA3.1-E2F1 and was negatively regulated by shcirc_0013731 in HCC cells (Fig. 2m-p). Finally, correlation analysis revealed positive correlation between the levels of circ_0013731 and E2F1 mRNA in HCC samples (N=45) (Fig. 2q). Therefore, E2F1 increased the expression of circ_0013731 through the binding to the MAN1A2 promoter.

\section{Overexpression of circ_0013731 promoted cell growth and inhibited ferroptosis}

The function of circ_0125310 was furtherly investigated. LV-circ_0013731 was transfected into HCC cells and the efficiency of LV-circ_0013731 was detected by qRT-PCR. Results showed that the expression level of circ_0013731 was successfully upregulated by LV- circ_0013731 in SMMC-7721 and QGY-7703 cells (Fig. 3a, b). EDU assays and colony formation assays indicated that LV-circ_0013731 enhanced the growth of SMMC-7721 and QGY-7703 cells (Fig. 3c-f). Then, erastin or RSL3 was used to induce ferroptosis of HCC cells. Results indicated that circ_0013731 overexpression weakening the effect of erastin or RSL3 on proliferation in SMMC-7721 and QGY-7703 cells (Fig. 3g, h). Moreover, LVcirc_0013731 reduced intracellular concentrations of iron and $\mathrm{Fe}^{2+}$ in SMMC-7721 and QGY-7703 cells after treating with erastin (Fig. 3i-I). Moreover, C11 BODIPY 581/591 staining coupled with flow cytometry analysis was performed. To validate the effect of circ_0013731 on ferroptosis, SMMC-7721 and QGY-7703 cells were transfected by sh-circ_0013731. HCC cells were treated by erastin and detected after 0 (red), 4 (green), 8 (orange), 12 (blue) hours. Lipid ROS showed an increased level after transfection with sh-circ_0013731 compared with that in negative control as shown in Fig. 3m, n. Thus, circ_0013731 promoted cell proliferation and inhibited ferroptosis in SMMC-7721 and QGY-7703 cells.

\section{miR-877-3p was the direct target of circ_0013731}


As shown in Fig. 4a, we consulted bioinformatics method using http://www.bioinf.com.cn/ and the putative binding sites of predicted miRNAs on circ_0013731 were shown. Luciferase activity of circ_0013731 in HCC cells transfected with five miRNA mimics was detected. Among the five candidates, the luciferase activity got the largest reduction compared with the negative control after transfection with mimic-miR-877-3p (Fig. 4b, c). Moreover, the luciferase activity had a largest raise compared with the negative control after transfection with inhibitor-miR-877-3p (Fig. 4d, e). Correlation analysis revealed negative correlation between the levels of circ_0013731 and miR-877-3p in HCC samples (N=45) (Fig. 4f). The expression levels of miR-877-3p in SMMC-7721 and QGY-7703 transfected with LV-circ_0013731 or sh-circ_0013731 were assessed. circ_0013731 was negatively regulated by circ_0013731 in SMMC-7721 and QGY-7703 (Fig. 4g-j). The direct binding sites between circ_0013731 and circ_0013731 were presented in Fig. 4k. The luciferase reporter activity of Wt-circ_0013731 in HCC cells co-transfected with miR-877-3p mimic was reduced while induced by the inhibitor. But, Mut-circ_0013731 could not be increased or decreased by mimic-miR-877-3p or inhibitor-miR-877-3p (Fig. 4l, m). Next, circ_0013731 and miR-877-3p in cell lysis were greatly pulled down and biotin-coupled miR-877-3p captured a fold change of circ_0013731 in the complex in SMMC-7721 and QGY-7703 (Fig. 4n, o). Afterwards, RIP was performed using Ago2 antibody in HCC cells transfected with miR-877-3p mimic or mimic NC. We proved that the enrichment of circ_0013731 was increased by circ_0013731 mimic (Fig. 4p, q). Therefore, miR-877-3p was the direct target of circ_0013731.

\section{Inhibition of miR-877-3p enhanced cell growth and inhibited ferroptosis.}

The function of miR-877-3p was furtherly explored. Inhibition-miR-877-3p was transfected into SMMC7721 and QGY-7703 cells and the efficiency of inhibition-miR-877-3p was detected by qRT-PCR. Results showed that the expression level of miR-877-3p was downregulated by inhibition-miR-877-3p in HCC cells (Fig. 5a, b). EDU assays and colony formation assays indicated that inhibition-miR-877-3p enhanced the growth of SMMC-7721 and QGY-7703 cells (Fig. 5c-f). After treated with erastin or RSL3, HCC cells were transfected with inhibition-miR-877-3p. Results indicated that inhibition-miR-877-3p decreased the erastin-induced or RSL3-induced growth inhibition of SMMC-7721 and QGY-7703 cells (Fig. $\mathbf{5 g}, \mathbf{~ h}$ ). Additionally, inhibition-miR-877-3p reduced intracellular concentrations of iron and $\mathrm{Fe}^{2+}$ in SMMC-7721 and QGY-7703 cells (Fig. 5i-I). Finally, lipid ROS was detected by C11 BODIPY 581/591 staining coupled with flow cytometry analysis. C11 BODIPY 581/591 staining coupled with flow cytometry analysis was performed. To validate the effect of miR-877-3p on ferroptosis, SMMC-7721 and QGY-7703 cells were transfected by mimic-miR-877-3p. HCC cells were treated by erastin and detected after 0 (red), 4 (green), 8 (orange), 12 (blue) hours. Results showed that lipid ROS showed an increased level after transfection with mimic-miR-877-3p in SMMC-7721 and QGY-7703 cells (Fig. 5m, n). Thus, inhibition of miR-877-3p enhanced HCC cell proliferation and suppressed ferroptosis. 


\section{circ_0013731 suppressed ferroptosis through miR-877-3p /SLC7A11axis}

Next, six genes predicted by Targetscan (http://www.targetscan.org/vert_72/) were screened in SMMC7721 and QGY-7703 transfected with inhibition-miR-877-3p. Among these candidates, SLC7A11 expression got the largest upregulation compared with the negative control after transfection with inhibition-miR-877-3p (Fig. 6a, b). Then, the protein expression of SLC7A11 in HCC cells was negatively modulated by inhibition-miR-877-3p (Fig. 6c, d). The direct binding sites predicted by Targetscan between miR-877-3p and SLC7A11 were presented in Fig. 6e. The luciferase reporter activity of Wt-SLC7A11 in HCC cells co-transfected with inhibition-miR-877-3p was upregulated, while Mut-SLC7A11 could not be increased by inhibitor-miR-877-3p (Fig. 6f, g). Correlation analysis revealed negative correlation between the levels of miR-877-3p and SLC7A11 in HCC samples ( $\mathrm{N}=45)$ (Fig. $6 \mathrm{~h}$ ). Then, the relationship between circ_0013731 and SLC7A11 was investigated. In Fig. 6i, the protein expression level of SLC7A11 was enhanced by LV- circ_0013731. Moreover, the luciferase reporter activity of Wt-SLC7A11 was increased by LV-circ_0013731, but Mut-SLC7A11 could not be increased by inhibitor-miR-877-3p (Fig. 6j, k). Then, SMMC-7721 and QGY-7703 were treated with LV-circ_0013731 or mimic-miR-877-3p. The protein expression level of SLC7A11 was induced by LV-circ_0013731, which could be reduced by mimic-miR877-3p (Fig. 6l, m). C11 BODIPY 581/591 staining coupled with flow cytometry analysis was performed. HCC cells were divided into four groups: LV-circ_0013731+mimic-NC (orange), LV-circ_0013731+ mimicmiR-877-3p (red), LV-NC+mimic.NC (green), LV-NC+ mimic-miR-877-3p (blue). As shown in Fig. $\mathbf{6 n}$ and Fig. 60, the lipid ROS expression level was decreased by LV-circ_0013731, which could be reduced by mimic-miR-877-3p. Then, intracellular concentrations of iron in HCC cells transfected with LVcirc_0013731 or mimic-miR-877-3p. Results showed that intracellular concentrations of iron were downregulated by LV-circ_0013731, which could be upregulated by mimic-miR-877-3p (Fig. 6p, q). Therefore, above results indicated that circ_0013731 inhibited ferroptosis through miR-877-3p /SLC7A11axis.

\section{circ_0013731 promoted HCC growth in vivo}

Then, in vivo assays were carried out to assess the effect of circ_0013731 on HCC progression. The xenograft tumor models of HCC were constructed by using SMMC-7721 and QGY-7703 cells infected with LV-circ_0013731, sh-circ_0013731 and negative control (every group=4). A picture of the xenograft is shown in Fig. 7a. The weight of the xenografts was calculated. The xenograft tumor was significantly heavier in the LV-circ_0013731 group than that in the LV-NC group (Fig. 7b). The xenograft tumor was significantly lighter in the sh-circ_0013731 group than that in the sh-NC group (Fig. 7c). The sizes of the xenograft in the LV-circ_0013731 and sh-circ_0013731 groups were calculated every 7 days.

circ_0013731 overexpression promoted tumor growth and circ_0013731 knockdown suppressed tumor growth (Fig. 7d-g). Moreover, the miR-877-3p /SLC7A11 axis was determined. The expression level of miR-877-3p was downregulated in the LV-circ_0013731 group, whereas that was upregulated in the shcirc_0013731 group. The expression level of SLC7A11 was upregulated in the LV-circ_0013731 group, 
whereas that was downregulated in the sh-circ_0013731 group (Fig. 7h-k). Moreover, the expression level of SLC7A11 was measured in each group tissues by IHC or IF assays. Results showed that SLC7A11 expression was upregulated in the LV-circ_0013731 group, whereas that was downregulated in the shcirc_0013731 group (Fig. 7l-o). Finally, a schematic diagram of mechanism of circ_0013731/ miR-877-3p /SLC7A11 in HCC progression was provided in Fig. 7p.

\section{Discussion}

In this study, we discovered that circ_0013731 was increased in the HCC tissues and HCC cell lines. circ_0013731 greatly promoted proliferation and suppressed ferroptosis in SMMC-7721 and QGY-7703 cells. Moreover, we found that E2F1 enhanced the expression of circ_0013731 through the binding to the circ_0013731 promoter. miR-877-3p was predicted as the target of circ_0013731. We verified the direct association between circ_0013731 and miR-877-3p. Inhibition of miR-877-3p enhanced HCC cell growth and inhibited ferroptosis. Then, SLC7A11 was predicted as the downstream target for miR-877-3p. circ_0013731 in HCC could promote growth and suppressed ferroptosis by sponging miR-877-3p and regulating SLC7A11 expression.

The occurrence and development of tumors is closely associated with deficiencies in programmed cell death $[24,25]$. Because of uncontrolled growth, tumor cells have a higher demand for iron than normal cells. Ferroptosis is the form of iron-dependent cell death, which is distinct from necroptosis and apoptosis [26, 27]. Thus, tumor cell is more susceptible to ferroptosis than normal cell because of abnormal iron metabolism [21, 28]. Increasing evidences have showed that ferroptosis acts important roles in HCC treatment. Ferroptosis is a vital part of mechanisms for sorafenib, which is first line therapy for HCC [29]. LDL-DHA nanoparticles, a potential new drug for HCC, cause ferroptosis in HCC tissues [30]. Thus, the role of circ_0013731 in ferroptosis of HCC captured our attention.

It has been elucidated that circ_0013731 expression is increased in HCC tissues and cells. Furtherly, the upstream regulators of circ_0013731 was investigated. Predicted by GCBI (https://www.gcbi.com.cn/gclib/html/index), E2F1, a vital transcription factor for circRNA, was chosen as the upstream regulators of circ_0013731. Our finding elucidated that E2 upregulated the circ_0013731 expression via binding to the circ_0013731 promoter. Then, overexpression of circ_0013731 in HCC inhibited cell growth and ferroptosis. Competing endogenous RNAs (ceRNA) is one of main molecular mechanisms for circRNA [31, 32]. Thus, the potential downstream miRNAs of circ_0013731 was investigated by bioinformatics method. miR-877-3p was confirmed as a target of circ_0013731. The role of miR-877-3p in HCC is unknown. We discovered that inhibition miR-877-3p could promote the HCC cell proliferation and repress the ferroptosis.

Solute carrier family 7 membrane 11 (SLC7A11) is a key coding gene of cystine/glutamate antiporter system xc- $[33,34]$. SLC7A11 promotes the synthesis of glutathione by mediating cystine uptake and glutamate release. Through the above way, SLC7A11 protects cells from oxidative stress, maintains the redox balance of cells, and prevents cell death induced by lipid peroxidation $[35,36]$. Iron regulates the 
transcription of SLC7A11 by ROS-Nrf2-ARE axis. It was confirmed that SLC7A11 could inhibit the cell iron death induced by iron overload in murine models [37]. SLC7A11 was confirmed as a target of miR-877-3p. Moreover, we discovered that the expression level of SLC7A11 was induced by circ_0013731, which could be reduced by mimic-miR-877-3p. Therefore, circ_0013731 up-regulated the expression level of SLC7A11 by sponging miR-877-3p in HCC cells. Finally, effect of circ_0013731 on HCC was detected in xenograft tumor models. Our data showed that circ_0013731 promoted HCC growth and regulated miR-877-3p/ SLC7A11 axis in vivo.

In this study, we further verified that circ_0013731 has oncogenic roles in HCC progression. Thus, our data discovered that circ_0013731/miR-877-3p/SLC7A11 regulatory loop may be potential therapeutic targets for HCC.

\section{Conclusion}

In summary, circ_0013731 is identified an important regulator in HCC. circ_0013731 is up-regulated by E2F1 and promoted cell growth in HCC. Furthermore, circ_0013731 suppresses the ferroptosis by regulation of miR-877-3p/SLC7A11 axis. Thus, circ_0013731 could be acted as potential therapeutical target for HCC.

\section{Declarations}

\section{Data Availability}

The data used to support the findings of this study are included within the article.

\section{Conflicts of Interest}

The authors declare that there is no conflict of interest regarding the publication of this paper.

\section{Funding Statement}

This work was supported by the National Key Research and Development projects intergovernmental cooperation in science and technology of China [grant number 2018YFE0126900]; the National Natural Science Foundation of China [grant numbers 82072025, 82072026]; the Science and Technology Project of Lishui City [grant numbers 2019GYX27, 2019SJZC61]; and The Medical and Health Science and Technology Project of Zhejiang Province [grant numbers 2020RC042, 2020KY380 and 2020KY1085].

\section{Acknowledgments}

Not applicable. 


\section{References}

1 Gad MAM, Eraky TE, Omar HM, et al. Role of real-time shear-wave elastogarphy in differentiating hepatocellular carcinoma from other hepatic focal lesions. Eur J Gastroenterol Hepatol, 2020.

2 Craig AJ, von Felden J, Garcia-Lezana T, et al. Tumour evolution in hepatocellular carcinoma. Nat Rev Gastroenterol Hepatol, 2020, 17(3): 139-152.

3 Yang JD, Hainaut P, Gores GJ, et al. A global view of hepatocellular carcinoma: trends, risk, prevention and management. Nat Rev Gastroenterol Hepatol, 2019, 16(10): 589-604.

4 Bruix J, da Fonseca LG, Reig M. Insights into the success and failure of systemic therapy for hepatocellular carcinoma. Nat Rev Gastroenterol Hepatol, 2019, 16(10): 617-630.

5 Kristensen LS, Andersen MS, Stagsted LVW, et al. The biogenesis, biology and characterization of circular RNAs. Nat Rev Genet, 2019, 20(11): 675-691.

6 Mehta SL, Dempsey RJ, Vemuganti R. Role of circular RNAs in brain development and CNS diseases. Prog Neurobiol, 2020, 186: 101746.

7 Zhou S, Wei J, Wang Y, et al. Cisplatin resistance-associated circRNA_101237 serves as a prognostic biomarker in hepatocellular carcinoma. Exp Ther Med, 2020, 19(4): 2733-2740.

8 Zhang X, Xu Y, Qian Z, et al. circRNA_104075 stimulates YAP-dependent tumorigenesis through the regulation of HNF4a and may serve as a diagnostic marker in hepatocellular carcinoma. Cell Death Dis, 2018, 9(11): 1091.

9 Yu J, Xu QG, Wang ZG, et al. Circular RNA cSMARCA5 inhibits growth and metastasis in hepatocellular carcinoma. J Hepatol, 2018, 68(6): 1214-1227.

10 Huang T, Yin L, Wu J, et al. MicroRNA-19b-3p regulates nasopharyngeal carcinoma radiosensitivity by targeting TNFAIP3/NF-kappaB axis. J Exp Clin Cancer Res, 2016, 35(1): 188.

11 Chipman LB, Pasquinelli AE. miRNA Targeting: Growing beyond the Seed. Trends Genet, 2019, 35(3): 215-222.

$12 \mathrm{Li}$ Z, Sun J, Liu W, et al. Changes in the circRNA expression profile of PC12 cells induced by TDCIPP exposure may regulate the downstream NF-kappaB pathway via the Traf2 gene. Chemosphere, 2020, 254 : 126834.

13 Abdollahzadeh R, Daraei A, Mansoori Y, et al. Competing endogenous RNA (ceRNA) cross talk and language in ceRNA regulatory networks: A new look at hallmarks of breast cancer. J Cell Physiol, 2019, 
234(7): 10080-10100.

14 Zhang PF, Wei CY, Huang XY, et al. Circular RNA circTRIM33-12 acts as the sponge of MicroRNA-191 to suppress hepatocellular carcinoma progression. Mol Cancer, 2019, 18(1): 105.

15 Han D, Li J, Wang H, et al. Circular RNA circMTO1 acts as the sponge of microRNA-9 to suppress hepatocellular carcinoma progression. Hepatology, 2017, 66(4): 1151-1164.

16 Yao Z, Xu R, Yuan L, et al. Circ_0001955 facilitates hepatocellular carcinoma (HCC) tumorigenesis by sponging miR-516a-5p to release TRAF6 and MAPK11. Cell Death Dis, 2019, 10(12): 945.

$17 \mathrm{Lu} \mathrm{J}$, Wang YH, Yoon C, et al. Circular RNA circ-RanGAP1 regulates VEGFA expression by targeting miR-877-3p to facilitate gastric cancer invasion and metastasis. Cancer Lett, 2020, 471: 38-48.

$18 \mathrm{Li} \mathrm{S}$, Zhu Y, Liang Z, et al. Up-regulation of $\mathrm{p} 16$ by miR-877-3p inhibits proliferation of bladder cancer. Oncotarget, 2016, 7(32): 51773-51783.

19 Kiruthiga C, Devi KP, Nabavi SM, et al. Autophagy: A Potential Therapeutic Target of Polyphenols in Hepatocellular Carcinoma. Cancers (Basel), 2020, 12(3).

20 Ozawa T, Maehara N, Kai T, et al. Dietary fructose-induced hepatocellular carcinoma development manifested in mice lacking apoptosis inhibitor of macrophage (AIM). Genes Cells, 2016, 21(12): 13201332.

21 Li J, Cao F, Yin HL, et al. Ferroptosis: past, present and future. Cell Death Dis, 2020, 11(2): 88.

22 Luo M, Wu L, Zhang K, et al. miR-137 regulates ferroptosis by targeting glutamine transporter SLC1A5 in melanoma. Cell Death Differ, 2018, 25(8): 1457-1472.

23 Wang M, Mao C, Ouyang L, et al. Long noncoding RNA LINC00336 inhibits ferroptosis in lung cancer by functioning as a competing endogenous RNA. Cell Death Differ, 2019, 26(11): 2329-2343.

24 Tummers B, Green DR. Caspase-8: regulating life and death. Immunol Rev, 2017, 277(1): 76-89.

$25 \mathrm{Lu}$ JV, Walsh CM. Programmed necrosis and autophagy in immune function. Immunol Rev, 2012, 249(1): 205-217.

26 Stockwell BR, Jiang X. The Chemistry and Biology of Ferroptosis. Cell Chem Biol, 2020, 27(4): 365375 .

27 Bayir H, Anthonymuthu TS, Tyurina YY, et al. Achieving Life through Death: Redox Biology of Lipid Peroxidation in Ferroptosis. Cell Chem Biol, 2020, 27(4): 387-408.

28 Han C, Liu Y, Dai R, et al. Ferroptosis and Its Potential Role in Human Diseases. Front Pharmacol, 2020, 11: 239. 
29 Werth EG, Rajbhandari P, Stockwell BR, et al. Time Course of Changes in Sorafenib-Treated Hepatocellular Carcinoma Cells Suggests Involvement of Phospho-Regulated Signaling in Ferroptosis Induction. Proteomics, 2020: e2000006.

30 Ou W, Mulik RS, Anwar A, et al. Low-density lipoprotein docosahexaenoic acid nanoparticles induce ferroptotic cell death in hepatocellular carcinoma. Free Radic Biol Med, 2017, 112: 597-607.

31 Huang $\mathrm{T}$, Huang $\mathrm{W}, \mathrm{Lu} \mathrm{H}$, et al. Identification and validation a TGF-beta-associated long non-coding RNA of head and neck squamous cell carcinoma by bioinformatics method. J Transl Med, 2018, 16(1): 46.

32 Abi A, Farahani N, Molavi G, et al. Circular RNAs: epigenetic regulators in cancerous and noncancerous skin diseases. Cancer Gene Ther, 2020, 27(5): 280-293.

33 Zhang L, Liu W, Liu F, et al. IMCA Induces Ferroptosis Mediated by SLC7A11 through the AMPK/mTOR Pathway in Colorectal Cancer. Oxid Med Cell Longev, 2020, 2020: 1675613.

34 Koppula P, Zhang Y, Zhuang $L$, et al. Amino acid transporter SLC7A11/xCT at the crossroads of regulating redox homeostasis and nutrient dependency of cancer. Cancer Commun (Lond), 2018, 38(1): 12.

35 Shi ZZ, Fan ZW, Chen YX, et al. Ferroptosis in Carcinoma: Regulatory Mechanisms and New Method for Cancer Therapy. Onco Targets Ther, 2019, 12: 11291-11304.

36 Yamaguchi I, Yoshimura SH, Katoh H. High cell density increases glioblastoma cell viability under glucose deprivation via degradation of the cystine/glutamate transporter xCT (SLC7A11). J Biol Chem, 2020, 295(20): 6936-6945.

37 Wang H, An P, Xie E, et al. Characterization of ferroptosis in murine models of hemochromatosis. Hepatology, 2017, 66(2): 449-465.

\section{Figures}


a

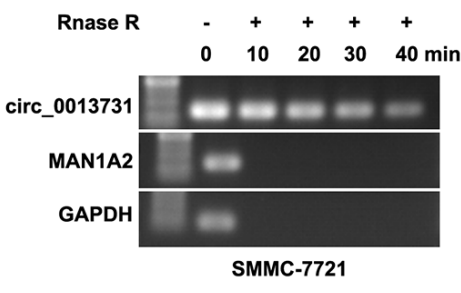

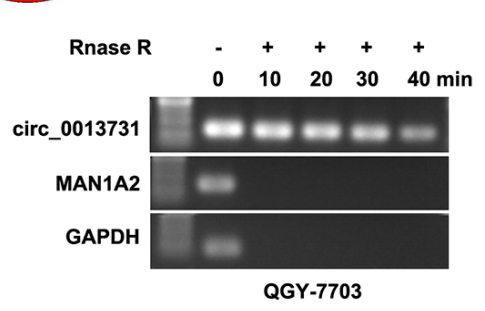

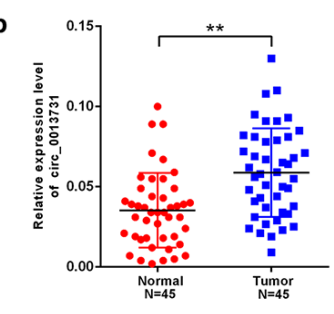

c
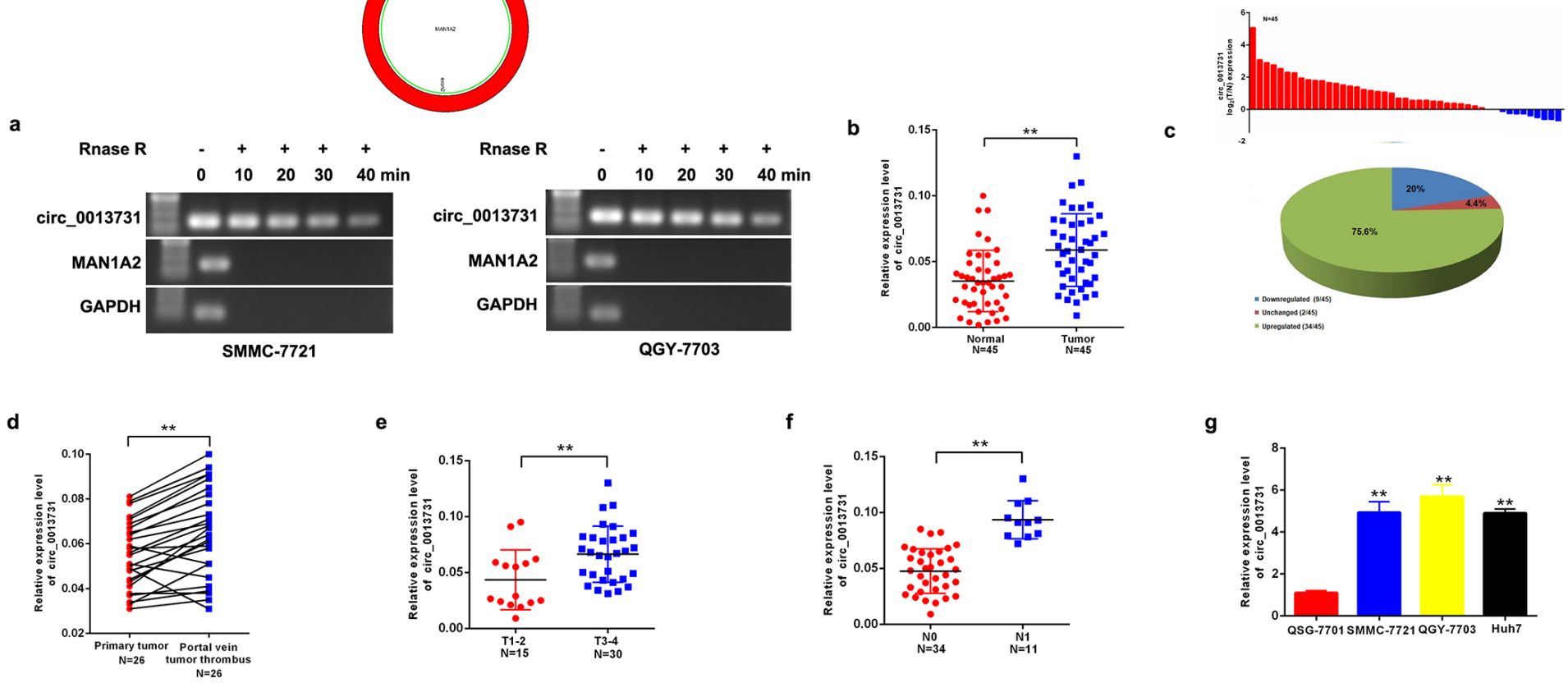

e

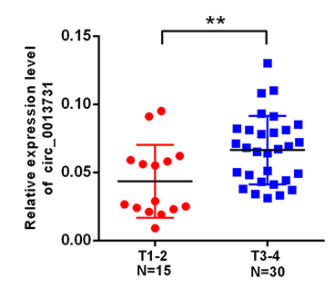

f

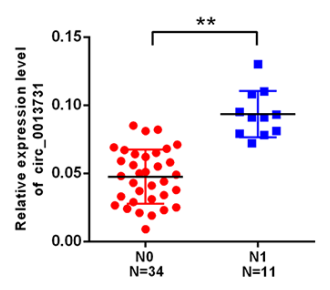

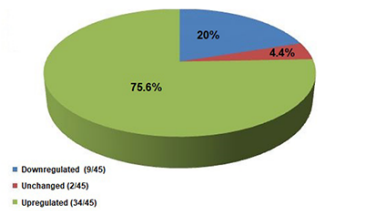

g

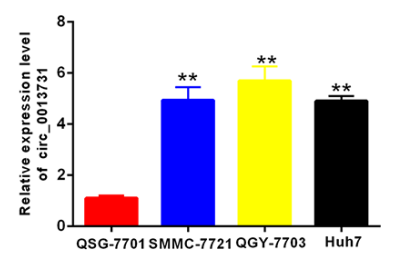

Figure 1

circ_0013731 was upregulated in hepatocellular carcinoma. a Validation of circ_0013731 in HCC cells by RNase R treatment and reverse transcription PCR analysis. b, c Left: expression levels of circ_0013731 in additional 45 paired samples of HCC were determined by qRT-PCR. Right: histogram and pie chart of the proportions of HCC samples in which circ_0013731 expression was downregulated (9/45, 20\%, blue), no change $(2 / 45,4.4 \%$, green), or upregulated $(34 / 45,75.6 \%$, red). d Expression levels of circ_0013731 in primary tumor and portal vein tumor thrombus $(\mathrm{N}=26)$. e Expression levels of circ_0013731 in T1-2 and T3-4 ( $N=45)$. $f$ Expression levels of circ_0013731 in N0 and N1 ( $N=45)$. g Expression levels of circ_0013731 in HCC cell lines. 
a

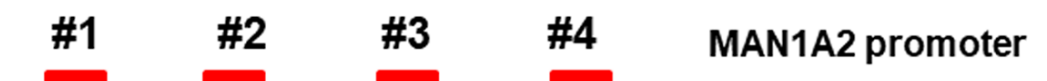

Wt MutWt Mut Wt Mut $\overparen{\text { Wt } \text { Mut }}$

Wt

\#1 TTTCGCGC \#2 TTAGGCGCGAGGG \#3 AGCGGCGCGAGGC \#4 CCTTCGCGCC

f

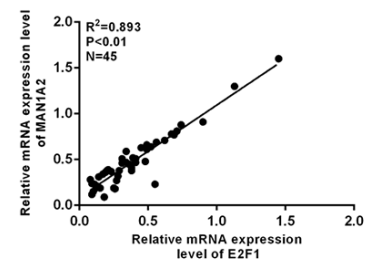

i

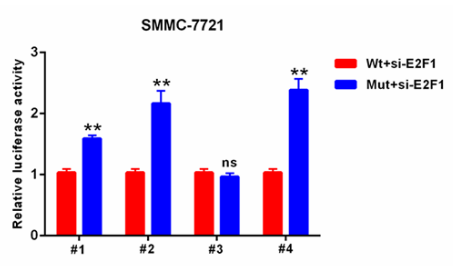

Mut

\#1 TTTCGCGC

\#2 TTAGGCGCGAGgG

\#3 AGcGgcGcGaGGC

\#4 CCTTCGGGCC

g

j

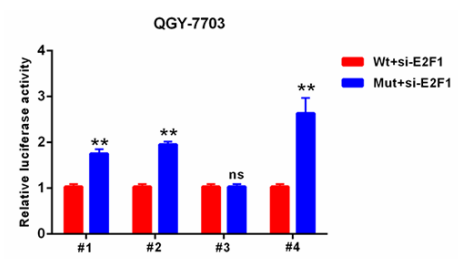

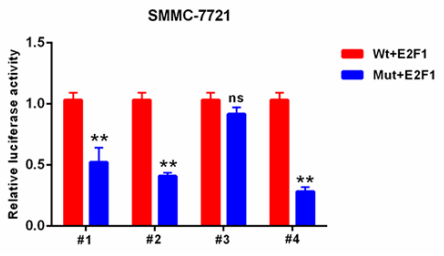

b

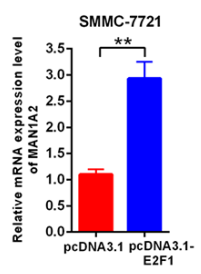

d

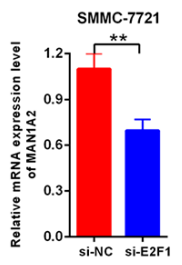

c

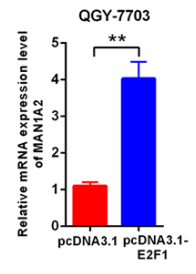

e

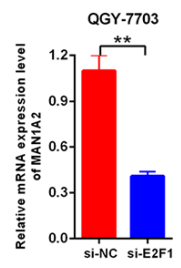

h

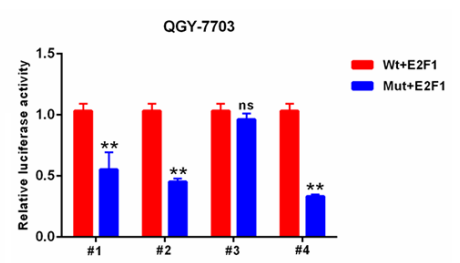

k

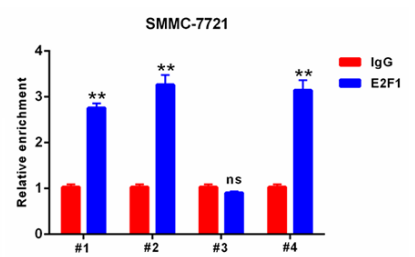

I

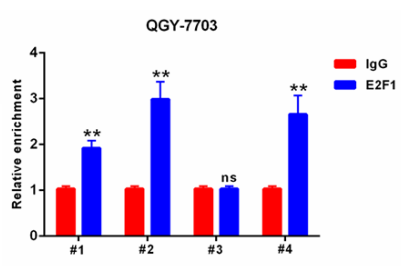

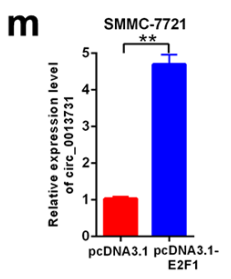

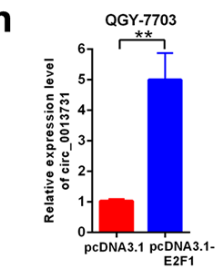

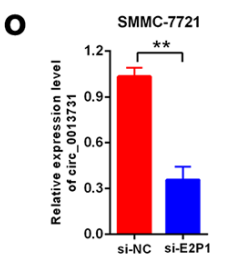
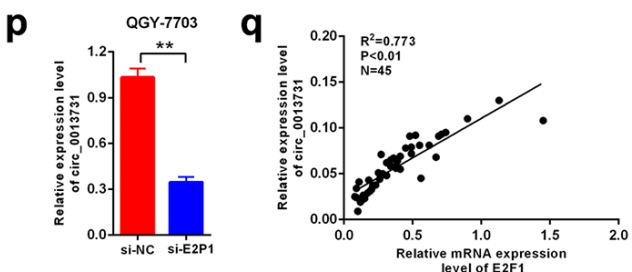

Figure 2

E2F1 enhanced the expression of circ_0013731 through the binding to the MAN1A2 promoter. a Schematic illustration of wild type (Wt) and mutant (Mut) sequences of four putative binding sites of E2F1 on MAN1A2 promoter are shown. b-e The expression of MAN1A2 was detected in SMMC-7721 and QGY-7703 transfected with E2F1 overexpression plasmids or siRNAs targeting E2F1 by qRT-PCR. $f$ Correlation analysis revealed positive correlation between the levels of MAN1A2 and E2F1 mRNA in the tumorous tissues of the $45 \mathrm{HCC}$ patients. g-j The relative luciferase activities were detected in SMMC7721 and QGY-7703 co-transfected with luciferase reporter plasmids containing wild type or mutant MAN1A2 promoter sequence and overexpression or siRNA of E2F1. k,l ChIP-qPCR assays were performed to determine which putative E2F1 binding site the MAN1A2 promoter was bound to in HCC cells, IgG was used as a negative control. m-p The circ_0013731 expression was valued in HCC cells transfected with E2F1 overexpression plasmids or si-E2F1 by qRT-PCR. q Correlation analysis revealed positive correlation between the levels of circ_0013731 and E2F1 mRNA in the tumorous tissues of the 45 HCC patients. 
a

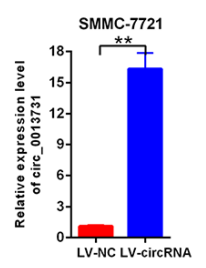

b

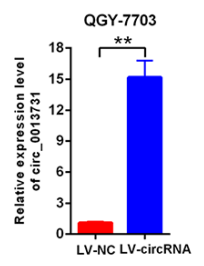

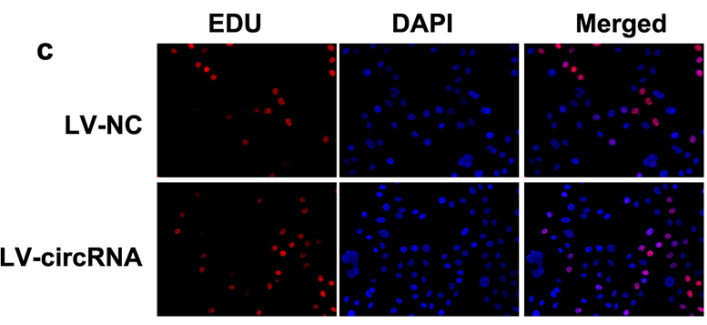

SMMC-7721

e

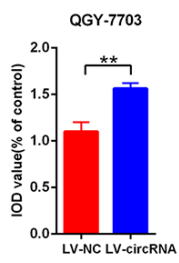

f

LV-NC

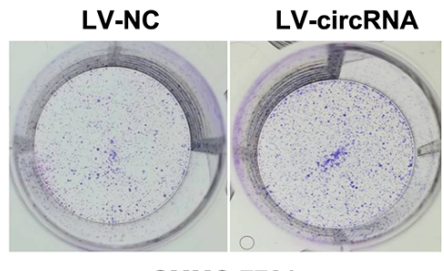

SMMC-7721

g

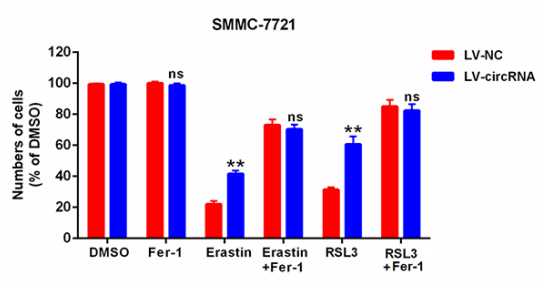

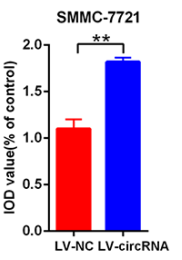

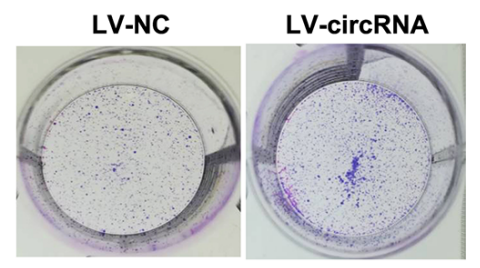

QGY-7703
QGY-7703

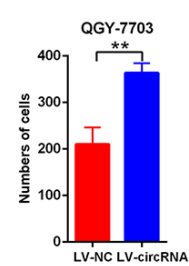

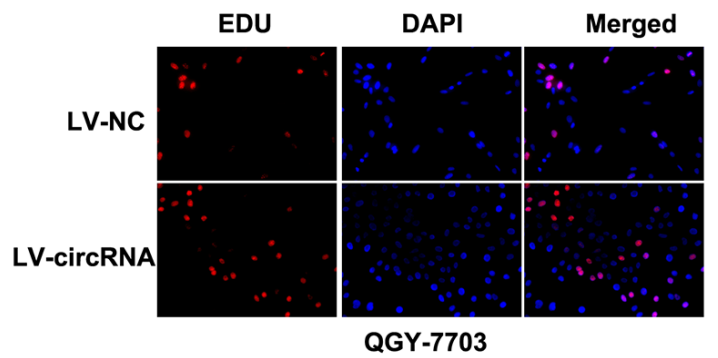

d

QGY-7703

h

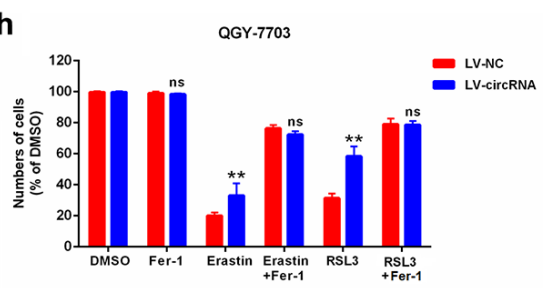

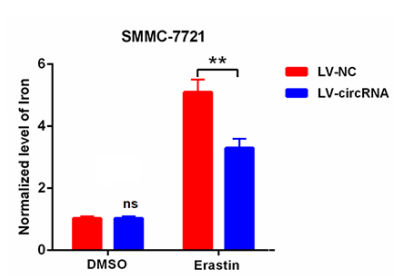

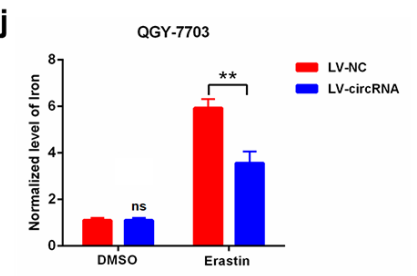

k

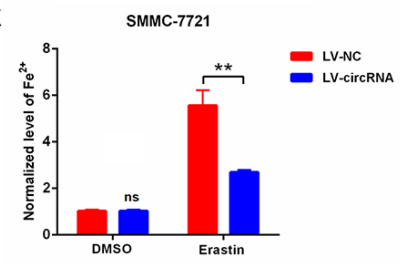

SMMC-7721
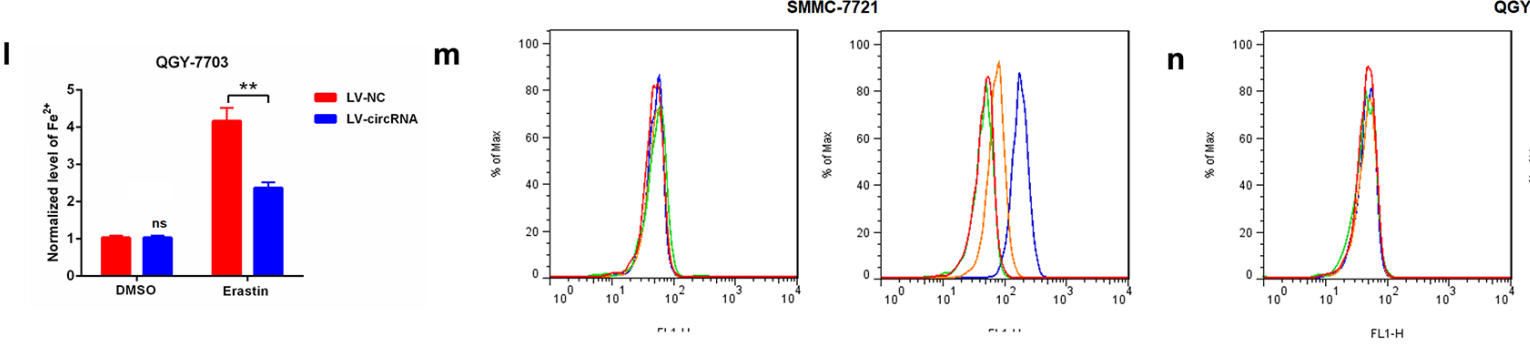

QGY-7703

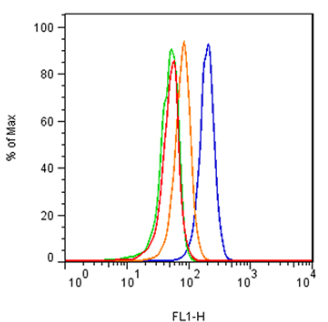

\section{Figure 3}

Overexpression of circ_0013731 promoted cell growth and inhibited ferroptosis. a,b qRT-PCR analysis was conducted to detect the level of LV-circ_0013731 in SMMC-7721 and QGY-7703. c,d Proliferating mesangial cells were labeled with EdU. HCC cells were treated with LV-circ_0013731. e,f Colony-formation assay of SMMC-7721 and QGY-7703 cells overexpressing circ_0013731. g,h MTT assays were used to analyze the responses of SMMC-7721 and QGY-7703 cells overexpressing circ_0013731 to ferrostatin $(2.0 \mu \mathrm{M})$, erastin $(10.0 \mu \mathrm{M})$, and RSL3 $(2.0 \mu \mathrm{M})$. $\mathrm{i}-\mathrm{I}$ Total iron $(\mathrm{i}, \mathrm{j})$, ferrous iron $(\mathrm{k}, \mathrm{l})$ were analyzed in HCC cells by overexpressing circ_0013731. m,n HCC cells transfected with sh-circ_0013731 were treated with 
erastin $(10 \mu \mathrm{M})$ for 0 (red), 4 (green), 8 (orange), 12 (blue) hours and the accumulation of lipid ROS was assessed by C11 BODIPY 581/591 staining coupled with flow cytometry analysis.

a

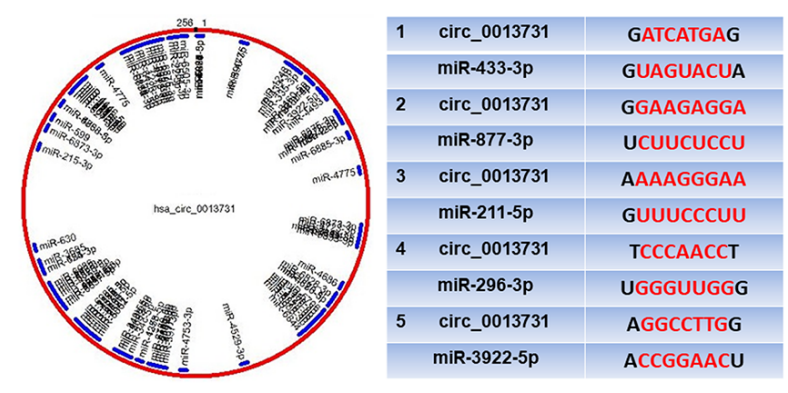

$\mathbf{f}$

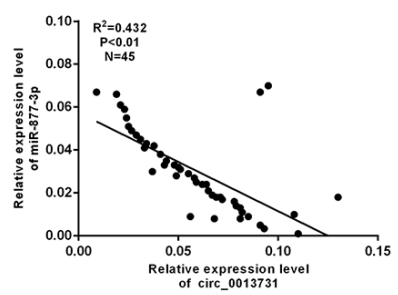

$\mathbf{k}$

3' GGAAGAGGA 5' Wt-circ_0013731

5' UCUUCUCCU 3' miR-877-3p

3' GGAAGAGGA 5' Mut-circ_0013731

n

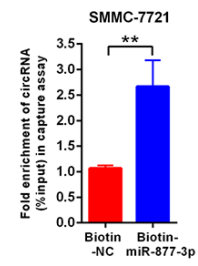

g

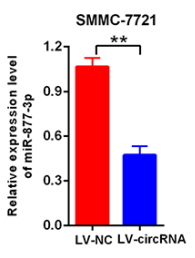

b

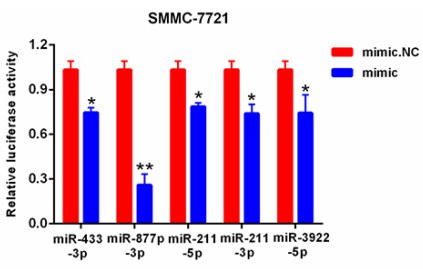

d

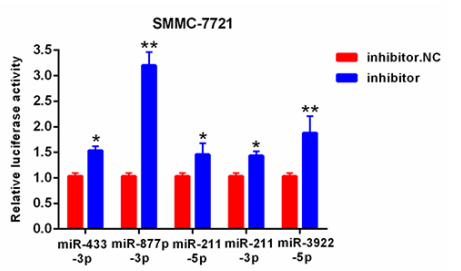

$\mathbf{h}$

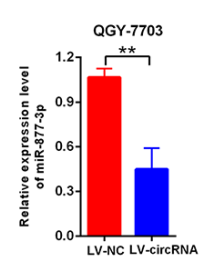

i

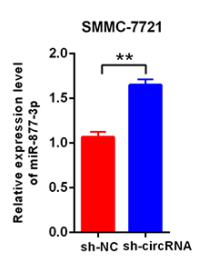

C

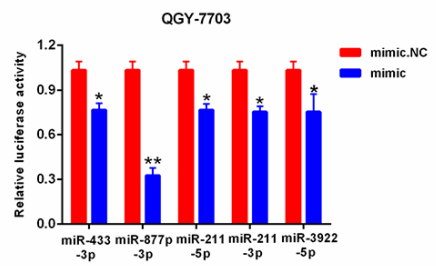

e

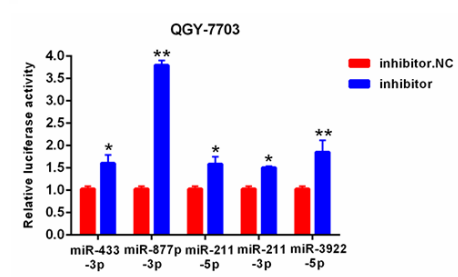

j

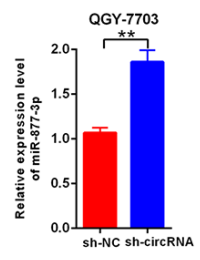

I
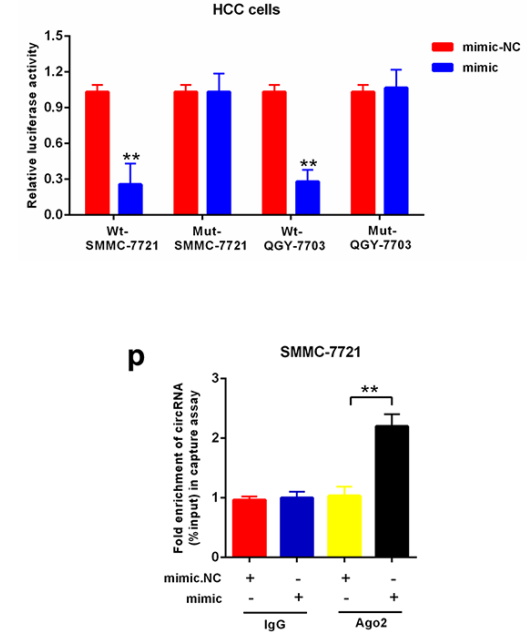

m

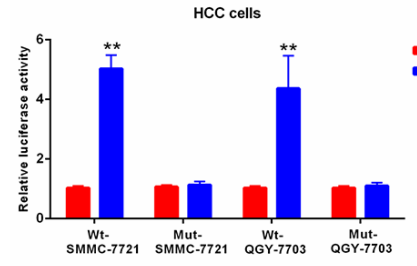

q

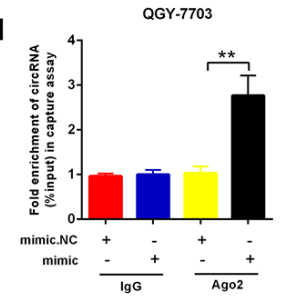

Figure 4

circ_0013731 functioned as a sponge for miR-877-3p. a Schematic illustration of sequences of five putative binding sites of circ_0013731 on potential target miRNAs are shown. b-e The relative luciferase activities were detected in SMMC-7721 and QGY-7703 co-transfected with luciferase reporter plasmids containing binding sites sequence and mimic or inhibitor. $f$ Correlation analysis revealed positive correlation between the levels of circ_0013731 and miR-877-3p in the tumorous tissues of the 45 HCC patients. g-j The expression of miR-877-3p was detected in SMMC-7721 and QGY-7703 transfected with LV-circ_0013731 or sh-circ_0013731 by qRT-PCR. $k$ The wild or mutant binding sites sequence of circ_0013731 and miR-877-3p were showed. I,m The relative luciferase activities were detected in HCC co- 
transfected with pGL3- circ_0013731-wt, pGL3-circ_0013731-mut or mimic-miR-877-3p. n,o RNA pulldown with a biotin-labeled circ_0013731 probe was implemented in HCC cells, followed by qRT-PCR and RT-PCR to test the enrichment of circ_0013731 and miR-877-3p. p,q RIP was performed using AGO2 antibody in SMMC-7721 and QGY-7703 transfected with miR-877-3p mimics or mimics NC, then the enrichment of circ_0013731 was detected.
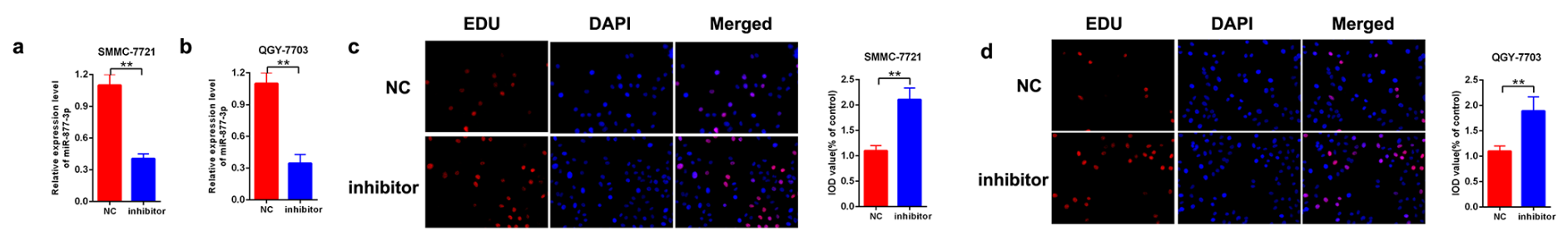

e
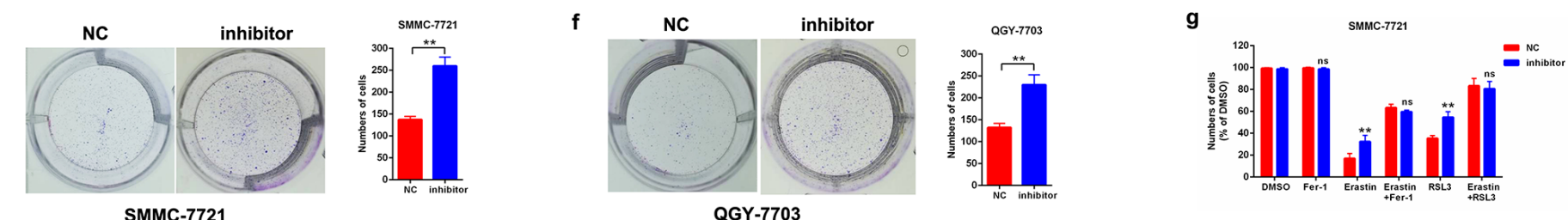

h
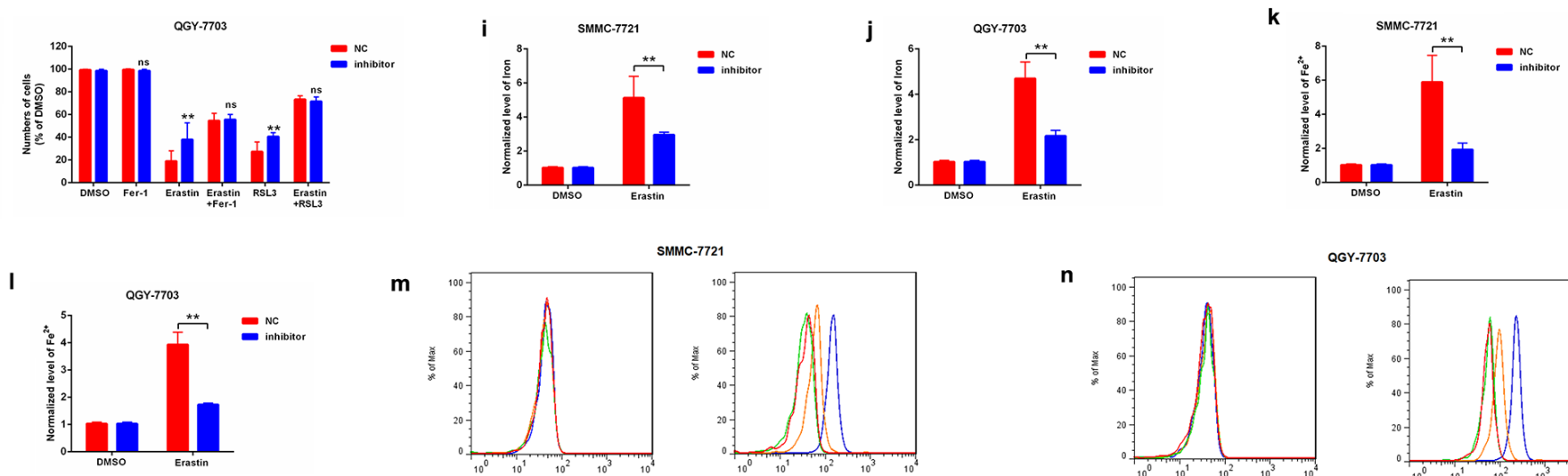

m

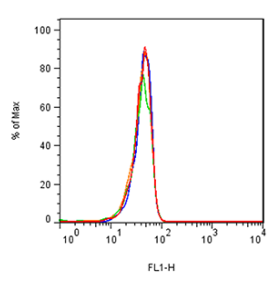

SMMC-7721

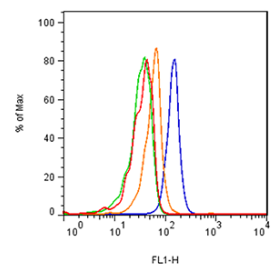

n

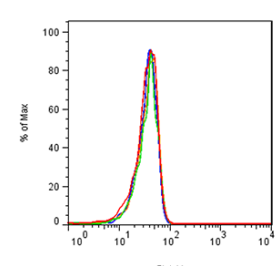

QGY-7703

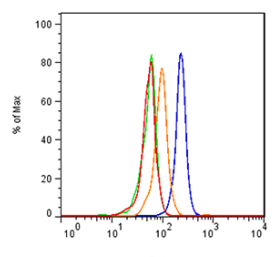

\section{Figure 5}

Inhibition of miR-877-3p enhanced cell growth and inhibited ferroptosis. a,b qRT-PCR analysis was conducted to detect the level of inhibitor-miR-877-3p in HCC cells. c,d Proliferating mesangial cells were labeled with EdU. HCC cells were treated with inhibitor-miR-877-3p. e, Colony-formation assay of SMMC7721 and QGY-7703 cells were treated with inhibitor-miR-877-3p. g,h MTT assays were used to analyze the responses of SMMC-7721 and QGY-7703 cells inhibitor-miR-877-3p to ferrostatin $(2.0 \mu \mathrm{M})$, erastin $(10.0 \mu \mathrm{M})$, and RSL3 $(2.0 \mu \mathrm{M})$. i- Total iron $(\mathrm{i}, \mathrm{j})$, ferrous iron $(\mathrm{k}, \mathrm{l})$ were analyzed in HCC cells by inhibition of miR-877-3p. m,n HCC cells transfected with mimic-miR-877-3p were treated with erastin $(10 \mu \mathrm{M})$ for 0 (red), 4 (green), 8 (orange), 12 (blue) hours and the accumulation of lipid ROS was assessed by $\mathrm{C} 11$ BODIPY 581/591 staining coupled with flow cytometry analysis. 

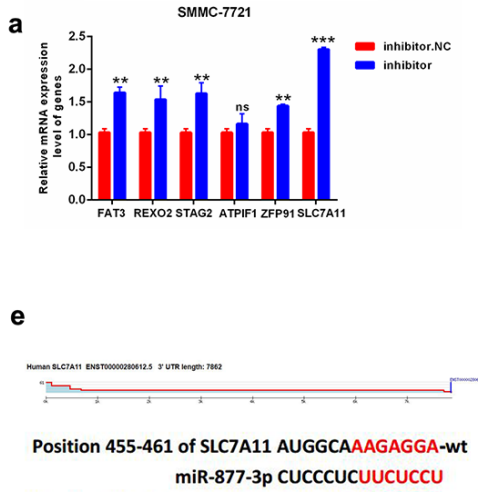
Position 455-461 of SLC7A11 AUGGCAAAGAGA-mut

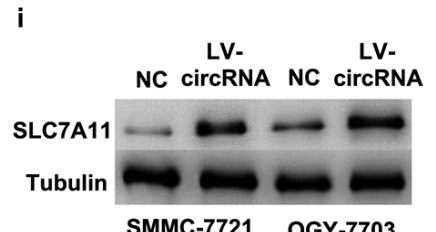

I
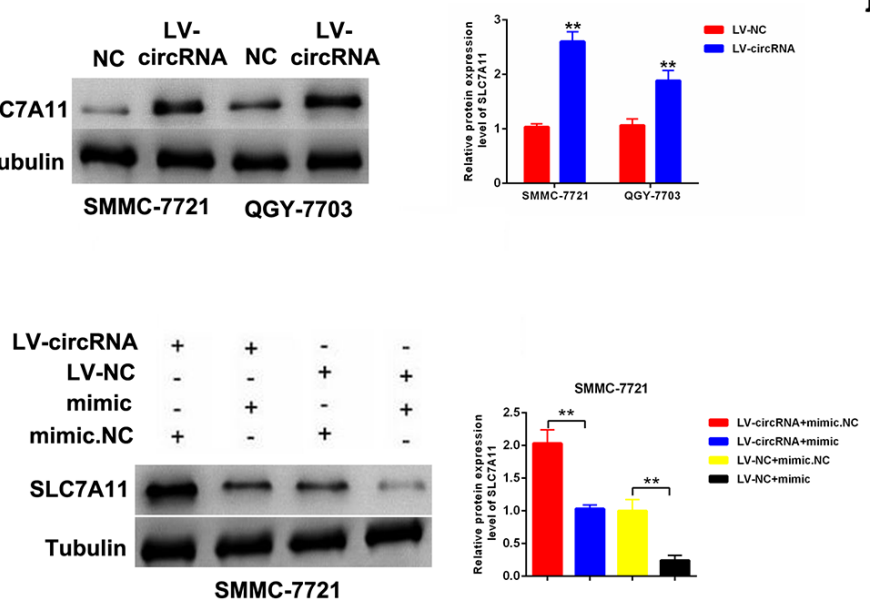

c

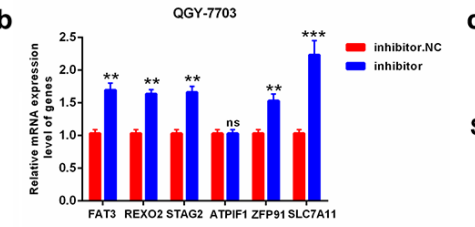

f

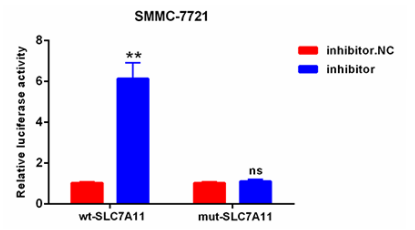

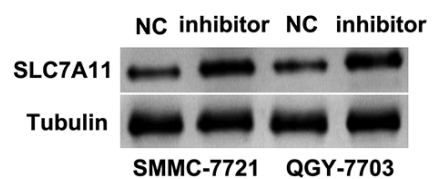

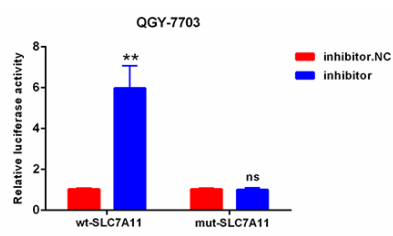

d

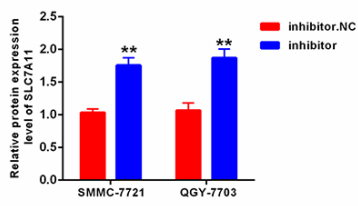

h

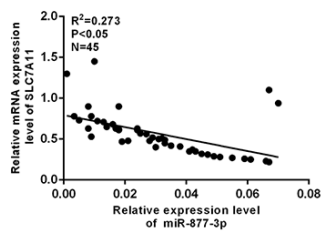

j

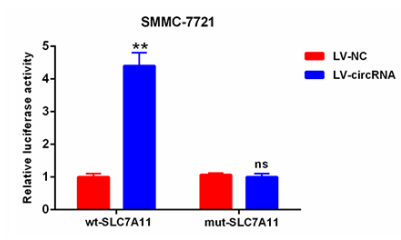

k

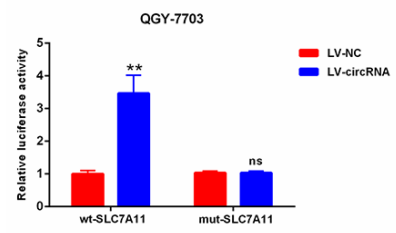

m
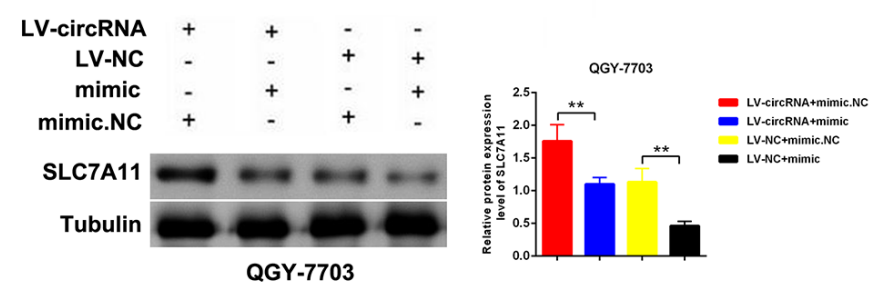

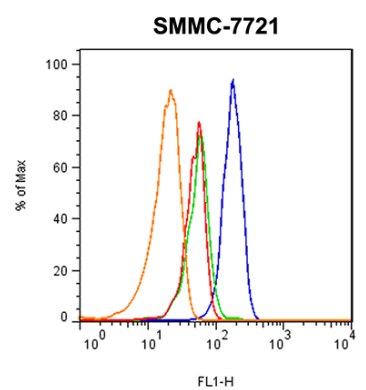

$\mathbf{0}$

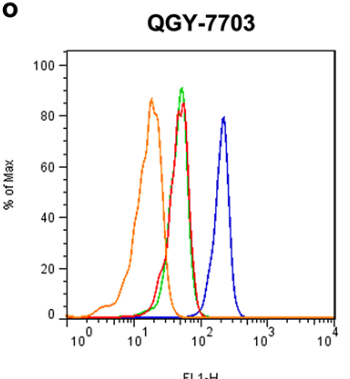

p

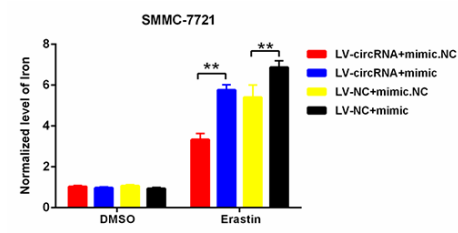

q

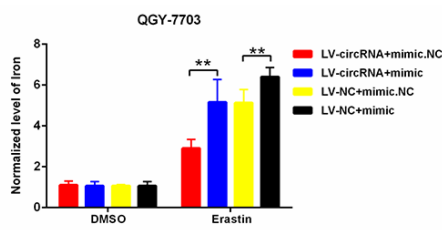

Figure 6

circ_0013731 suppressed ferroptosis through miR-877-3p /SLC7A11axis. a,b Six potential target genes of miR-877-3p were screened by qRT-PCR in SMMC-7721 and QGY-7703 transfected by inhibitor-miR-877-3p. c,d Western blot was conducted to determine the expression of SLC7A11 in HCC cells after transfection with inhibitor-miR-877-3p. e The wild or mutant binding sites sequence of SLC7A11 and miR-877-3p were shown. $f, g$ The relative luciferase activities were detected in HCC cells co-transfected with pGL3SLC7A11-wt, pGL3-SLC7A11-mut or inhibitor-miR-877-3p. h Correlation analysis revealed positive correlation between the levels of miR-877-3p and SLC7A11 HCC tissues. i The protein expression level of SLC7A11 was detected in SMMC-7721 and QGY-7703 transfected by LV-circ_0013731. j,k The relative luciferase activities were detected in SMMC-7721 and QGY-7703 co-transfected with pGL3- SLC7A11-wt, 
pGL3-SLC7A11-mut, or circ_0013731. I,m HCC cells were transfected by LV-circ_0013731 or mimic-miR877-3p. The protein expression level of SLC7A11 was detected in SMMC-7721 and QGY-7703 transfected. n,o HCC cells were divided into four groups: LV-circ_0013731+mimic-NC (orange), LV-circ_0013731+ mimic-miR-877-3p (red), LV-NC+mimic.NC (green), LV-NC+ mimic-miR-877-3p (blue). C11 BODIPY $581 / 591$ staining was performed. HCC cells treated by erastin $(10 \mu \mathrm{M})$ were co-transfected by LVcirc_0013731 or mimic-miR-877-3p. p.q Ferrous iron was detected in SMMC-7721 and QGY-7703 cotransfected with LV-circ_0013731 or mimic-miR-877-3p.
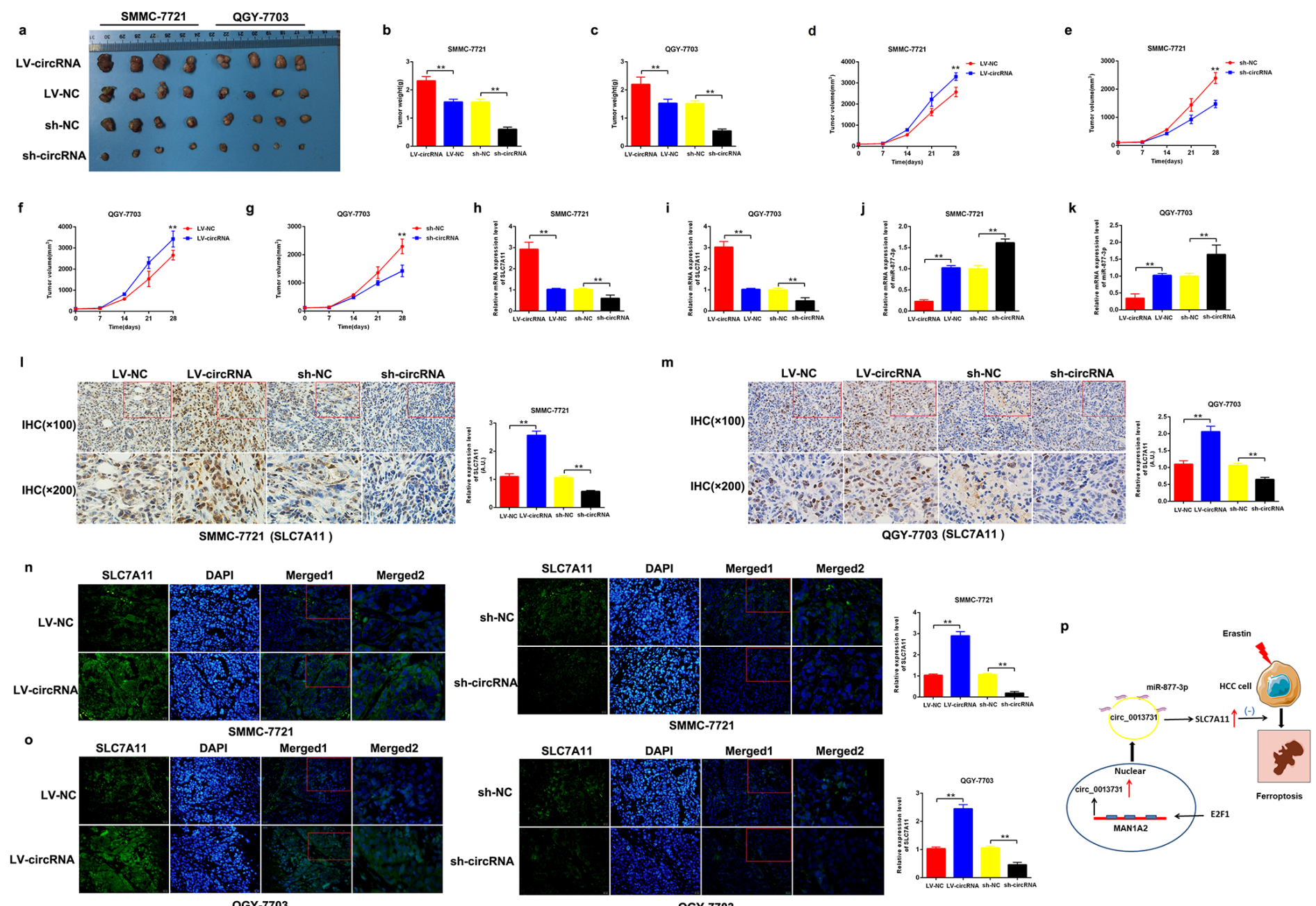

\section{Figure 7}

circ_0013731 facilitated HCC growth in vivo. a The representative images of xenograft tumor in each group were displayed ( $\mathrm{N}=4)$. b,c Tumor weight of was analyzed in each group. $\mathrm{d}$-g Tumor volumes were measured once a week in each group. $h$,i The expression level of SLC7A11 was measured in each group tissues by qRT-PCR analysis. j,k The expression level of miR-877-3p was measured in each group. I-o The expression level of SLC7A11 was measured in each group tissues by IHC or IF assays. $p$ Schematic diagram of mechanism on this research was showed. 